\title{
On the set of extreme core allocations for minimal cost spanning tree problem
}

\author{
Christian Trudeau (University of Windsor) \\ Juan Vidal-Puga (Universidade de Vigo)
}

Working paper $15-05$

Working papers are in draft form. This working paper is distributed for purposes of comment and discussion only. It may not be reproduced without permission of the copyright holder. Copies of working papers are available from the author or at http://ideas.repec.org/s/wis/wpaper.html. 


\title{
On the set of extreme core allocations for minimal cost spanning tree problems*
}

\author{
Christian Trudeau ${ }^{\dagger} \quad$ Juan Vidal-Puga ${ }^{\ddagger}$
}

November 30, 2015

\begin{abstract}
Minimal cost spanning tree problems connect agents efficiently to a source when agents are located at different points and the cost of using an edge is fixed. We propose a method, based on the concept of marginal games, to generate all extreme points of the corresponding core. We show that three of the most famous solutions to share the cost of mcst problems, the Bird, folk and cycle-complete solutions, are closely related to our method.

Keywords: Minimal cost spanning tree problems, extreme core allocations, reduced game, Bird solution, folk solution, cycle-complete solution.
\end{abstract}

JEL Classifications: C71, D63

*The first author acknowledges the financial support of the Social Sciences and Humanities Research Council of Canada (ref. 435-2014-0140). The second author acknowledges support of the Ministerio de Ciencia e Innovacion (ref. ECO2011-23460) and Ministerio de Economia y Competitividad (ref. ECO2014-52616-R).

${ }^{\dagger}$ Department of Economics, University of Windsor 401 Sunset Avenue, Windsor, Ontario, Canada. Email: trudeauc@uwindsor.ca

${ }^{\ddagger}$ Research Group of Economic Analysis and Departamento de Estatistica e IO. Universidade de Vigo. 36200 Vigo (Pontevedra), Spain. Email: vidalpuga@uvigo.es 


\section{Introduction}

Minimal cost spanning tree (mcst) problems model a situation where agents are located at different points and need to be connected to a source in order to obtain a good or information. Agents do not care if they are connected directly to the source or indirectly through other agents. The cost to build a link between two agents or an agent and the source is a fixed number, meaning that the cost is the same whether one or ten agents use that particular link. Mcst problems can be used to model various real-life problems, from telephone and cable TV to water supply networks.

The core of mcst problems has been and early focus of attention, with Bird (1976) and Granot and Huberman (1981) showing that it is always nonempty and Granot and Huberman (1984) providing an algorithm to generate multiple core allocations. We present an improvement over these results by providing a method that allows to obtain the full set of extreme core allocations.

The method is based on the concept of marginal games (Núñez and Rafels, 1998), in which we assign an agent her marginal cost to join the grand coalition, remove her from the problem and update the stand-alone costs of the remaining coalitions: they can either keep their original stand-alone cost or the stand-alone cost of them with the departing player, net of her cost share. This reduction is itself a special case of the Davis-Maschler reduction (Davis and Maschler, 1965). Given an ordering of the agents, repeating the process until all players are removed allows to find an extreme core allocation.

This method or very similar ones have been implemented for the assignment problem (Núñez and Rafels, 2003) and shortest path problems (Bahel and Trudeau, 2014), among others ${ }^{1}$. In the non-cooperative setting, a similar approach consists in ordering buyers according to a given permutation and letting them buy goods in that order (Pérez-Castrillo and Sotomayor, 2002; Vidal-Puga, 2004).

The method does not work as well on all problems. Even though there exist sufficient conditions for the method to always generate the full set of extreme core allocations (Potters et al., 1989; Driessen, 1988; Núñez and Rafels, 1998), none of them are satisfied, in general, by mcst problems. We are still

\footnotetext{
${ }^{1}$ A related approach is that of Tijs (2005); Funaki et al. (2007); Kongo et al. (2010); Tijs et al. (2011), who also look for extreme core allocations given some lexicographic order. However, their approach is explicitly based on the core constraints and not on marginal games.
} 
able to prove that the method generates the full set of extreme core allocations, using a representation of marginal games as minimal cost spanning tree problems with priced nodes. This new problem is a generalization of both mcst problems and Steiner tree problems (Hwang and Richards, 1992; Skorin-Kapov, 1995).

By taking the average of these extreme core allocations for all permutations, we obtain a very natural cost sharing solution, identified as the selective value in Vidal-Puga (2004) and that coincides with the so-called Average lexicographic value, or Alexia (Tijs, 2005). If the game is concave, it also corresponds to the Shapley value. We show that our procedure is very close to three well-known cost-sharing solutions for mcst problems.

Firstly, if we only consider permutations that correspond to the order in which we connect agents in an optimal network configuration, we obtain directly the Bird solution (Bird, 1976). The Bird solution was the first solution to be shown to always be in the core and it is known for its simplicity, as we may assign cost at the same time as we construct an optimal tree.

Secondly, we show that for elementary problems (where all costs are either 0 or 1), our solution corresponds to the cycle-complete solution (Trudeau, 2012). The cycle-complete solution is obtained by modifying the cost of some links before taking the Shapley value of the corresponding cost game: we reduce the cost of edge $(i, j)$ if there exists a cycle that goes through nodes $i$ and $j$ and such that its most expensive edge is cheaper than the direct edge $(i, j)$. The modification is enough to make the corresponding cost game concave, and thus the Shapley value stable.

Thirdly, we show that for elementary problems, our solution applied to the public version of the mcst problem (where a coalition can use the nodes of its neighbors to connect to the source) corresponds to the folk solution (Feltkamp et al., 1994; Bergantiños and Vidal-Puga, 2007). The folk solution is obtained in the same way as the cycle-complete solution, but we look at paths instead of cycles.

An interpretation of our result is that for elementary problems, the folk solution is the permutation-weighted average of extreme core allocations of the public game, while the cycle-complete solution is the permutation-weighted average of extreme core allocations of the private game (where a coalition can only build a network among the nodes of its members).

The paper is divided as follows: In Section 2 we define the minimal cost spanning tree problems. In Section 3 we describe our method and show that it generates extreme core allocations. In Section 4 we show that it generates 
the full set of extreme core allocations. In Section 5 we explore some links with popular cost sharing solutions. Section 6 contains some discussions. Proofs and counter-examples are in appendix.

\section{The model}

A (cost sharing) game is a pair $(N, C)$ where $N=\{1, \ldots, n\}$ is a nonempty, finite set of agents, and $C$ is a characteristic function that assigns to each nonempty coalition $S \subseteq N$ a nonnegative cost $C(S) \in \mathbb{R}_{+}$that represents the price agents in $S$ should pay in order to receive a service. In particular, we assume that the agents in $N$ need to be connected to a source, denoted by 0 . Let $N_{0}=N \cup\{0\}$. For any set $Z$, define $Z^{p}$ as the set of all nonordered pairs $(i, j)$ of elements of $Z$. In our context, any element $(i, j)$ of $Z^{p}$ represents the edge between nodes $i$ and $j$. Let $c=\left(c_{e}\right)_{e \in N_{0}^{p}}$ be a vector in $\mathbb{R}_{+}^{N_{0}^{p}}$ with $N_{0}^{p}=\left(N_{0}\right)^{p}$ and $c_{e}$ representing the cost of edge $e$. Given $E \subset N_{0}^{p}$, its associated cost is $c(E)=\sum_{e \in E} c_{e}$.

Let $\Gamma$ be the set of all cost vectors. Since $c$ assigns cost to all edges $e$, we often abuse language and call $c$ a cost matrix. A minimal cost spanning tree problem is a triple $(0, N, c)$. Since 0 and $N$ do not change, we omit them in the following and simply identify a mcst problem $(0, N, c)$ by its cost matrix c.

Given $l \in N_{0}$, a cycle $p_{l l}$ is a set of $K \geq 3$ edges $\left(i_{k}, i_{k+1}\right)$, with $k \in$ $\{0, \ldots, K-1\}$ and such that $i_{0}=i_{K}=l$ and $i_{1}, \ldots, i_{K-1}$ distinct and different than $l$. Given $l, m \in N_{0}$, a path $p_{l m}$ between $l$ and $m$ is a set of $K$ edges $\left(i_{k}, i_{k+1}\right)$, with $k \in\{0, \ldots, K-1\}$, containing no cycle and such that $i_{0}=l$ and $i_{K}=m$. Let $P_{l m}\left(N_{0}\right)$ be the set of all such paths between nodes $l$ and $m$.

A spanning tree is a non-orientated graph without cycles that connects all elements of $N_{0}$. A spanning tree $t$ is identified by the set of its edges.

We call mcst a spanning tree that has a minimal cost. It can be obtained using Prim's algorithm, which has $|N|$ steps. First, pick an edge $(0, i)$ such that $c_{0 i} \geq c_{0 j}$ for all $j \in N$. We then say that $i$ is connected. In the second step, we choose an edge with the smallest cost connecting an agent in $N \backslash\{i\}$ either directly to the source or to $i$, which is connected. We continue until all agents are connected, at each step connecting an agent not already connected to an agent already connected or to the source. Note that the mest might not be unique. Let $t^{*}(c)$ be a mest and $\mathcal{T}^{*}(c)$ be the set of all mest for the 
cost matrix $c$. Let $C(N, c)$ be the cost of a mcst. Let $c^{S}$ be the restriction of the cost matrix $c$ to the coalition $S_{0} \subseteq N_{0}$. Let $C(S, c)$ be the cost of the mcst of the problem $\left(S, c^{S}\right)$. Given these definitions, we say that $C$ is the stand-alone cost function associated with $c$.

For any $S \subseteq N$, let $x(S)=\sum_{i \in S} x_{i}$. An allocation is a vector $x \in \mathbb{R}^{N}$ such that $x(N)=C(N)$. Given $S \subseteq N$ and $x \in \mathbb{R}^{N}$, we denote as $x_{S} \in \mathbb{R}^{S}$ the restriction of $x$ to $\mathbb{R}^{S}$.

For any cost matrix $c$, the associated cost game is given by $(N, C)$ with $C(S)=C(S, c)$ for all $S \subseteq N$. We then say that $C$ is a mest game. We define the set of stable allocations as $\operatorname{Core}(C)$. Formally, an allocation $x \in \operatorname{Core}(C)$ if $x(S) \leq C(S)$ for all $S \subseteq N$.

\section{A method to find extreme core allocations}

Our method to find extreme core allocations is based on the concept of marginal games of Núñez and Rafels (1998), but we define it in terms of a closely related source connection problem. A mcst problem with priced nodes is a tupla $(N, P, y, c)$ where $P \subseteq N$ are nodes that do not need to be connected and $y \in \mathbb{R}^{P}$ is the vector whose coordinates are the prices that nodes in $P$ pay to agents in $N \backslash P$ if they are actually connected. Nodes in $P$ are called priced nodes. Hence, the cost of $(N, P, y, c)$ is defined as

$$
C(N, P, y, c)=\min _{T \subseteq P}\{C((N \backslash P) \cup T)-y(T)\}
$$

and the cost of a subset $S \subseteq N \backslash P$ is given by

$$
C(S, P, y, c)=\min _{T \subseteq P}\{C(S \cup T)-y(T)\} .
$$

In particular, $C(N, P, y, c)=C(N \backslash P, P, y, c)$.

As usual, the core of a mest problem with priced nodes $(N, P, y, c)$ is the set of allocations $x \in \mathbb{R}^{N \backslash P}$ that satisfy $x(N \backslash P)=C(N, P, y, c)$ and $x(S) \leq C(S, P, y, c)$ for all $S \subseteq N \backslash P$.

Notice that mest problems with priced nodes generalize both mcst problems (when $P=\emptyset$ ) and minimal cost Steiner tree problems (when $y_{i}=0$ for all $i \in P$ ).

For simplicity, when $y \in \mathbb{R}^{N}$, we write $(N, P, y, c)$ instead of $\left(N, P, y_{P}, c\right)$.

Let $\Pi$ be the set of orders of $N$. Let $\pi=\left(\pi_{1}, \ldots, \pi_{n}\right) \in \Pi$. Given $\pi \in \Pi$ and $i \in N$, let $P^{\pi i}=\left\{\pi_{i}, \ldots, \pi_{n}\right\}$ be the set of nodes that come 
after $\pi_{i}$ (including $\pi_{i}$ ) in the order $\pi$. For notational convenience, we denote $P^{\pi n+1}=\emptyset$. We define the reduced marginal cost vector of $C$ related to permutation $\pi$, denoted as $y^{r \pi}(C)$, or simply $y^{r \pi}$, in a recursive manner, starting with $y_{\pi_{n}}^{r \pi}$ and making our way down to $y_{\pi_{1}}^{r \pi}$ :

$$
y_{\pi_{i}}^{r \pi}=C\left(\left\{\pi_{1} \ldots \pi_{i}\right\}, P^{\pi i+1}, y^{r \pi}, c\right)-C\left(\left\{\pi_{1} \ldots \pi_{i-1}\right\}, P^{\pi i+1}, y^{r \pi}, c\right)
$$

for $i=n, \ldots, 1$.

It is not difficult to check that we are applying the marginal games idea of Núñez and Rafels (1998). After removing players, we allow coalitions of remaining players to either keep their original stand-alone cost or take the stand-alone cost they had with (some of) the departing players, net of their assigned cost shares. In our interpretation with priced nodes, this means that a coalition has to decide to build a network that includes or not some of these priced nodes, consisting of the set of departed players.

Example 1 Let $N=\{1,2,3,4\}$ and $c$ be as described in the following ( $i$ horizontally, $j$ vertically) and illustrated in Figure 1:

$\begin{array}{ccccc}c_{i j} & 1 & 2 & 3 & 4 \\ 0 & 1 & 6 & 5 & 5 \\ 1 & & 6 & 4 & 2 \\ 2 & & & 5 & 5 \\ 3 & & & & 5\end{array}$

Let $\pi=[1,2,3,4]$. We can see that $C(N, c)=12$ and $C(\{1,2,3\}, c)=$ 10 , yielding $y_{4}^{r \pi}=2$. Agent 4 then becomes a priced node. Now, we have $C\left(\{1,2,3\},\{4\}, y^{r \pi}, c\right)=10$ (as there's no advantage to connect agent 4 and collect 2) and $C\left(\{1,2\},\{4\}, y^{r \pi}, c\right)=6$ (as connecting through agent 4 is advantageous), yielding $y_{3}^{r \pi}=4$. We then have both agents 3 and 4 as priced nodes. We have $C\left(\{1,2\},\{3,4\}, y^{r \pi}, c\right)=6$ and $C\left(\{1\},\{3,4\}, y^{r \pi}, c\right)=1$ and thus $y_{2}^{r \pi}=5$. Finally, we have $C\left(\{1\},\{2,3,4\}, y^{r \pi}, c\right)=1=y_{1}^{r \pi}$. We thus obtain $y^{r \pi}=(1,5,4,2)$.

Núñez and Rafels (1998) provide a sufficient condition for $y^{r \pi}$ to be an extreme point of the core, and in fact for the set $\left\{y^{r \pi}\right\}_{\pi \in \Pi}$ to be the set of extreme points of the core. The sufficient condition is that of almostconcavity of the cost game: $C(S)+C(T) \geq C(S \cup T)+C(S \cap T)$ for all $S, T \subset N$ such that $S \cup T \neq N$. We thus have all concavity conditions except those involving the grand coalition. We show in appendix that the condition is not satisfied by games generated by most problems.

Nevertheless, we show that our method provides core allocations. 


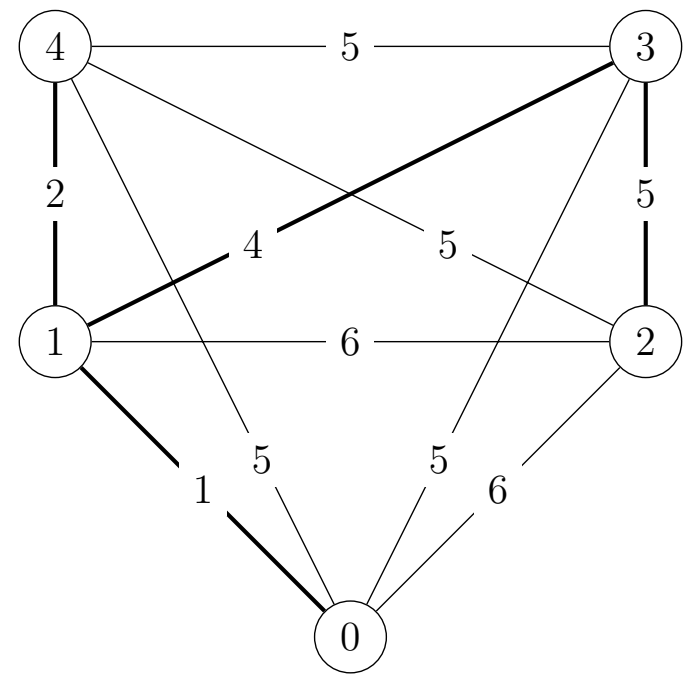

Figure 1: Example of a minimal cost spanning tree problem.

Proposition 1 For any mcst game $C$, any permutation $\pi \in \Pi$, and any $i \in N \cup\{n+1\}, y_{N \backslash P^{\pi i}}^{r \pi}$ belongs to the core of $\left(N, P^{\pi i}, y^{r \pi}, c\right)$ and, moreover, any $t^{*} \in \mathcal{T}^{*}(c)$ is an optimal tree in $\left(N, P^{\pi i}, y^{r \pi}, c\right)$.

The result $y^{r \pi} \in \operatorname{Core}(C)$ arises as the particular case when $i=n+1$.

\section{The set of extreme core allocations}

We now have shown that our procedure generates allocations in the core. By definition, they are also extreme core allocations, defined as follows:

Definition 1 An allocation $y \in \operatorname{Core}(C)$ is an extreme core allocation if there do not exist $y^{\prime}, y^{\prime \prime} \in \operatorname{Core}(C), y^{\prime} \neq y^{\prime \prime}$ and $\lambda \in(0,1)$ such that $y=$ $\lambda y^{\prime}+(1-\lambda) y^{\prime \prime}$.

We denote the set of extreme core allocations as ExtCore $(C)$. Funaki et al. (2007) define a leximal as the lexicographic minimum of the core with respect to a given order. ${ }^{2}$ It is not difficult to see that our allocation $y^{r \pi}$ corresponds to the leximal with respect to the reverse order of $\pi$.

\footnotetext{
${ }^{2}$ Funaki et al. (2007) define the leximal as the lexicographic maximum of the core of a value game. The lexicographic minimum of the core is conceptually equivalent for cost games.
} 
The convex hull of $\left\{y^{r \pi}\right\}_{\pi \in \Pi}$ is thus the convex hull of the leximals, defined by Funaki et al. (2007) as the lexicore. By definition, the lexicore is a subset of the core. A sufficient condition for the lexicore to be equal to the core is for the corresponding exact game (where all coalitions can attain their stand-alone cost in the core) to be concave. We show in the appendix that this condition does not hold generally for mest problems.

Nevertheless, we next show that the core allocations defined in the previous section actually constitute the whole set of extreme core allocations. We proceed by first showing that for any coalition, we attain the maximal allocation compatible with the core. We need to prove two intermediary results before getting to the main result of this section.

First, we define the alternative stand-alone cost $\widehat{C}(S)$, where we let $S$ pick a partner $T$ and compute the sum of the costs of $S$ with $T$ and $N \backslash T$, to which we subtract the cost of the grand coalition.

Formally, for all $c \in \Gamma$, let $(N, \widehat{C})$ be defined in the following way:

Step 0: $\widehat{C}(S)=C(S)$ for all $S$ such that $|S| \geq n-1$.

Step $k: \widehat{C}(S)=\min \left\{C(S), \min _{\emptyset \neq T \subset N \backslash S} \widehat{C}(S \cup T)+\widehat{C}(N \backslash T)-\widehat{C}(N)\right\}$ for all $S$ such that $|S|=n-1-k$, for $k=1, \ldots, n-2$.

Step $n-1: \widehat{C}(\emptyset)=0$.

Hence,

$$
\widehat{C}(S)=\min _{T \subseteq N \backslash S}\{\widehat{C}(S \cup T)+\widehat{C}(N \backslash T)-C(N)\}
$$

for all $S \subseteq N$.

The cost function $\widehat{C}$ has the same core as $C$ and a coalition $S$ cannot receive more than $\widehat{C}(S)$ in any core allocation.

Lemma 1 For all $c \in \Gamma$, Core $(\widehat{C})=\operatorname{Core}(C)$.

Our next step is to show that for any $S$, there exists at least one permutation $\pi$ such that $y^{r \pi}(S)=\widehat{C}(S)$. To do so, we once again use the mest with priced nodes representation.

We know (Proposition 1) that $y_{N \backslash P^{\pi i}}^{r \pi}$ belongs to the core of $\left(N, P^{\pi i}, y^{r \pi}, c\right)$ and, moreover, that any $t^{*} \in \mathcal{T}^{*}(c)$ is an optimal tree in any $\left(N, P^{\pi i}, y^{r \pi}, c\right)$.

Let $t^{*} \in \mathcal{T}^{*}(c)$. We denote $t^{*}=\left\{\left(i, i^{*}\right)\right\}_{i \in N}$, where $i^{*}$ is the predecessor of node $i$ in $t^{*}$, i.e. $i^{*}$ is the adjacent node to node $i$ in the (unique) path in $t^{*}$ from node $i$ to the source. 
We then define $i \preceq_{*} j$ as the partial relation in $N$ given by " $i$ precedes $j$ in $t^{* \prime}$, that is, $i \preceq_{*} j$ iff $j \in F^{* i}$, where $F^{* i}$ is the set of followers of node $i$ in $t^{*}$ (including node $i$ ).

Given a tree $t$, we denote as $t(N) \subseteq N$ the set of nodes that connect to the source through $t$.

The next lemma will be needed in our main result. See Figure 2 for a graphical interpretation.

Lemma 2 Let $\left(N, P^{a}, y^{r \pi}, c\right)$ and $\left(N, P^{b}, y^{r \pi}, c\right)$ be two mcstp with priced nodes and assume there exists $P_{1} \subseteq P^{a} \cap P^{b}$ such that $P_{1}$ is a maximal subset of both $P^{a}$ and $P^{b}$ whose nodes are connected in $t^{*}$ for some $t^{*} \in$ $\mathcal{T}^{*}(c)$. For any $p_{1} \in P_{1}$, there exists a tree $t^{1}$ with $N \backslash P_{1} \subseteq t^{1}(N) \subseteq$ $N \backslash\left\{p_{1}\right\}$ such that $t^{1}$ is optimal in both $\left(N \backslash\left\{p_{1}\right\}, P^{a} \backslash\left\{p_{1}\right\}, y^{r \pi}, c\right)$ and $\left(N \backslash\left\{p_{1}\right\}, P^{b} \backslash\left\{p_{1}\right\}, y^{r \pi}, c\right)$.
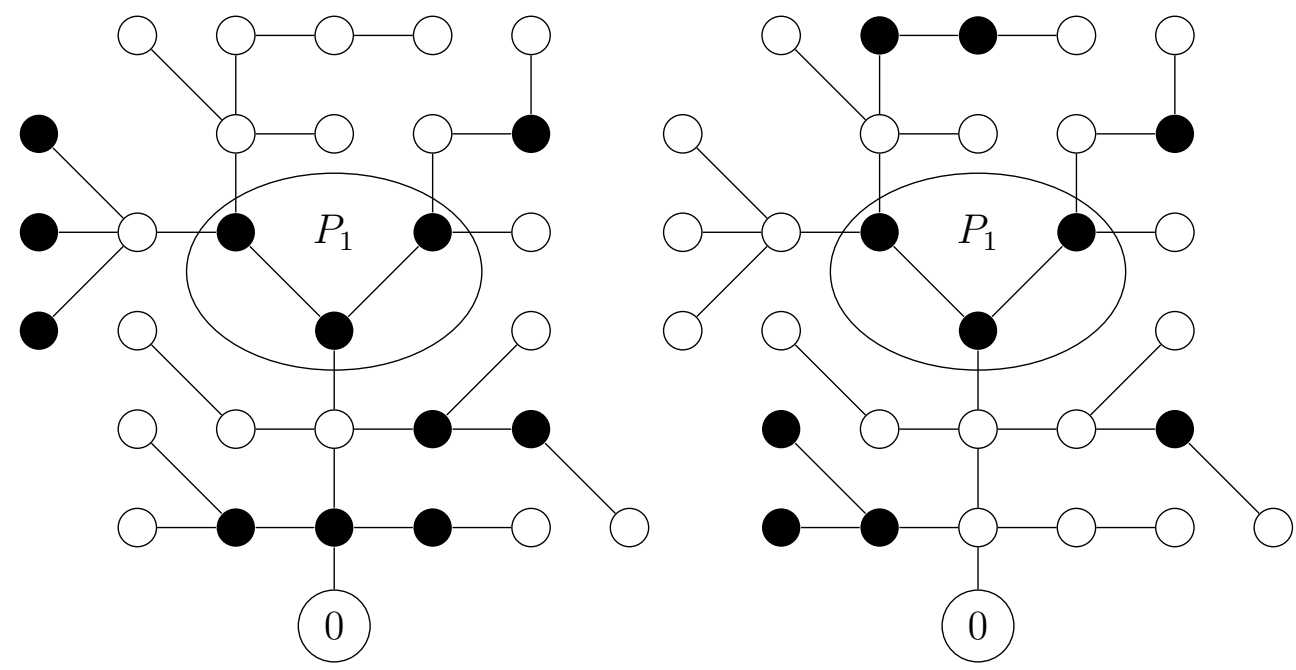

Figure 2: Two most problems with priced nodes and the same cost matrix. Priced nodes are those in black. $P_{1}$ is a maximal set of priced nodes in both problems. Lemma 2 says that, if we remove some node in $P_{1}$, then we can find a common optimal tree for both reduced problems.

Given $P \subseteq N$ and $\pi \in \Pi$, we say that $\pi$ is $t^{*}$-compatible with $P$ if the following two conditions hold: 
External $t^{*}$-compatibility Nodes in $S=N \backslash P$ that follow or immediately precede in $P$ a node in $t^{*}$ come first in $\pi$ :

$$
\left.\begin{array}{l}
\pi_{i} \preceq_{*} \pi_{j} \text { or } \pi_{i}^{*}=\pi_{j} \\
\pi_{i} \in P \\
\pi_{j} \in S
\end{array}\right\} \Longrightarrow j<i .
$$

Internal $t^{*}$-compatibility Nodes inside $P$ follow in $\pi$ the partial order $\preceq_{*}$ :

$$
\left.\begin{array}{l}
\pi_{i} \preceq_{*} \pi_{j} \\
\pi_{i}, \pi_{j} \in P
\end{array}\right\} \Longrightarrow i \leq j
$$

It is clear that there always exists an order $t^{*}$-compatible with $P$. For example, let $\pi^{*} \in \Pi$ be an order obtained by Prim's algorithm, where nodes that are closer to the source in $t^{*}$ come first. Clearly, this order satisfies internal $t^{*}$-compatibility. We then move the nodes in $P$ to the last positions, maintaining the internal order in $P$ and $N \backslash P$ unaffected. The resulting order $\pi^{* P}$ satisfies both internal and external $t^{*}$-compatibility.

Example 2 We revisit Example 1, with $P=\{1,3,4\}$.

Take $t^{*}=\{(0,1),(1,4),(1,3),(3,2)\}$. External $t^{*}$-compatibility implies that nodes 1 and 3 should go after node 2 . Internal $t^{*}$-compatibility implies that nodes 3 and 4 should go after node 1 . These two conditions together give two unique $t^{*}$-compatible orders: $[2,1,4,3]$ and $[2,1,3,4]$. In both cases, $y^{r \pi}=(0,6,4,2)$.

The same happens if we take $t^{*}=\{(0,1),(1,3),(1,4),(4,2)\}$ instead.

We say that $\pi$ is $c$-compatible with $P$ if there exists some $t \in \mathcal{T}^{*}(c)$ such that $\pi$ is $t$-compatible with $P$.

In the previous example, $y^{r \pi}(\{2\})=\widehat{C}(\{2\})=6$ for each $\pi$-compatible with $\{1,3,4\}$. We now show that this holds in general.

Proposition 2 Given $S \subseteq N$, we have $y^{r \pi}(S)=\widehat{C}(S)$ for all $\pi \in \Pi$ order c-compatible with $N \backslash S$.

The next proposition will allow us to deduce the main result.

Proposition 3 For all $c \in \Gamma, \operatorname{ExtCore}(C)=\left\{y^{r \pi}\right\}_{\pi \in \Pi}$. 
As a direct implication of Proposition 3, we obtain our main result: a complete description of the core.

Theorem 1 For all $c \in \Gamma$, Core $(C)$ is the convex hull of $\left\{y^{r \pi}\right\}_{\pi \in \Pi \text {. }}$

We conclude this section by providing the full set of extreme points of the core for our running example.

Example 3 Consider the mcst problem illustrated in Example 1. The table below shows the 6 distinct extreme points we obtain with our method. $|\pi|$ indicates the number of permutations that yield the given extreme point.

$\begin{array}{ccccc}|\pi| & x_{1} & x_{2} & x_{3} & x_{4} \\ 8 & -3 & 5 & 5 & 5 \\ 6 & 1 & 5 & 4 & 2 \\ 3 & -2 & 5 & 4 & 5 \\ 3 & 0 & 6 & 4 & 2 \\ 3 & 0 & 5 & 5 & 2 \\ 1 & -2 & 6 & 4 & 4\end{array}$

\section{$5 \quad$ Links with the core of the public game and with known cost sharing solutions}

In this section, we show that our method allows to bridge many gaps in the mest literature.

First, we consider a variant of the mcst problem, called the public mcst problem, where a coalition can use all nodes, including those belonging to agents outside of the coalition, to connect to the source. We show that the core of the public mcst problem is easily obtained from the result of the previous section.

Second, we can define a new cost-sharing solution as the permutationweighted average of extreme core allocations. We show that it has close links with three of the most famous cost sharing solutions in the literature on mcst.

\subsection{The core of the public mest problem}

The public mcst problem allows a coalition $S$ to connect to the source using the nodes of agents in $N \backslash S$. We thus obtain the following characteristic cost 
function. For all $S \subseteq N$, we have

$$
C^{P u b}(S, c)=\min _{T \subseteq N \backslash S} C(S \cup T, c) .
$$

While most of the literature uses the private version of the game, the distinction between the two games has been noted in Bogomolnaia and Moulin (2010) and Trudeau (2013). We obtain the following result.

Theorem 2 For all $c \in \Gamma, \operatorname{Core}\left(C^{P u b}(N, c)\right)=\operatorname{Core}(C(N, c)) \cap \mathbb{R}_{+}^{N}$.

Thus, restricting $\operatorname{Core}(C)$ to its elements in $\mathbb{R}_{+}^{N}$ yields $\operatorname{Core}\left(C^{P u b}(N, c)\right)$. Therefore, once we have the extreme points of $\operatorname{Core}(C(N, c))$, we have a trivial way to obtain the extreme points of $\operatorname{Core}\left(C^{P u b}(N, c)\right)$.

Example 4 Consider the mcst problem illustrated in Example 1. The table below shows the 4 distinct extreme points we obtain for Core $\left(C^{P u b}\right)$.

$\begin{array}{llll}x_{1} & x_{2} & x_{3} & x_{4}\end{array}$

$\begin{array}{llll}1 & 5 & 4 & 2\end{array}$

$\begin{array}{llll}0 & 6 & 4 & 2\end{array}$

$\begin{array}{llll}0 & 5 & 5 & 2\end{array}$

$\begin{array}{llll}0 & 5 & 4 & 3\end{array}$

Notice that the first three extreme allocations are the extreme allocations of Example 3 that are in $\mathbb{R}_{+}^{N}$. The fourth allocation is on the line between the extreme core allocations (of $C)(-2,5,4,5)$ and $(1,5,4,2)$, at the intersection with $\mathbb{R}_{+}^{N}$.

We complete this subsection by showing how to extend the definition of $y^{r \pi}$ to the public most game.

A public mcst problem with priced nodes is a tupla $(N, P, y, c)$ where $P \subseteq N$ are nodes that do not need to be connected and $y \in \mathbb{R}_{+}^{P}$ is the vector whose coordinates are the prices that nodes in $P$ pay to agents in $N \backslash P$ if they are actually connected. In the public mcst problem with priced nodes, a coalition $S \subset N \backslash P$ can use the nodes of agents in $N \backslash(S \cup P)$ to connect without paying anything, while it collects the payoff in $y$ if it connects through the nodes of agents in $P$. Hence, the cost of $(N, P, y, c)$ is defined as

$$
C^{P u b}(N, P, y, c)=\min _{T \subseteq P}\{C((N \backslash P) \cup T)-y(T)\}
$$


and the cost of a subset $S \subseteq N \backslash P$ is given by

$$
C^{P u b}(S, P, y, c)=\min _{T \subseteq N \backslash S}\{C(S \cup T)-y(T \cap P)\} .
$$

In particular, $C^{P u b}(N, P, y, c)=C^{P u b}(N \backslash P, P, y, c)$. We define the reduced marginal cost vector of $C^{P u b}$ related to permutation $\pi$, denoted as $y^{r \pi}\left(C^{P u b}\right)$ in a recursive manner, starting with $y_{\pi_{n}}^{r \pi}\left(C^{P u b}\right)$ and making our way down to $y_{\pi_{1}}^{r \pi}\left(C^{P u b}\right): y_{\pi_{i}}^{r \pi}\left(C^{P u b}\right)$

$$
=C^{P u b}\left(\left\{\pi_{1} \ldots \pi_{i}\right\}, P^{\pi i+1}, y^{r \pi}, c\right)-C^{P u b}\left(\left\{\pi_{1} \ldots \pi_{i-1}\right\}, P^{\pi i+1}, y^{r \pi}, c\right) .
$$

Notice that, since $C^{P u b}$ is a monotonically increasing function, $y_{\pi_{n}}^{r \pi}\left(C^{P u b}\right) \geq$ 0 . By the definition of the public mcst problem with priced nodes, the fact that $y_{\pi_{n}}^{r \pi}\left(C^{P u b}\right) \geq 0$ guarantees that $y_{\pi_{n-1}}^{r \pi}\left(C^{P u b}\right) \geq 0$. Applying the argument successively, we obtain that $y^{r \pi}\left(C^{P u b}\right) \in \mathbb{R}_{+}^{N}$.

Proposition 4 For all $c \in \Gamma$, ExtCore $\left(C^{P u b}\right)=\left\{y^{r \pi}\left(C^{P u b}\right)\right\}_{\pi \in \Pi}$.

The proof is identical to the proof of Proposition 3 and is omitted.

\subsection{Links with well-known cost-sharing solutions}

A cost sharing solution (or rule) assigns a cost allocation $y(c)$ to any admissible cost matrix $c$. We start by building a solution from the allocations defined in the previous sections. This solution is the selective value (VidalPuga, 2004) defined as the average of the reduced marginal cost vectors, i.e.

$$
y^{s}(C)=\sum_{\pi \in \Pi(N)} \frac{1}{n !} y^{r \pi}(C) .
$$

By our previous section, $y^{s}(C)$ is the permutation-weighted average of the extreme allocations of $\operatorname{Core}(C)$. It is also corresponds to the Alexia value, as the permutation-weighted average of leximals (Tijs, 2005; Funaki et al., 2007; Kongo et al., 2010; Tijs et al., 2011). We define $y^{s}\left(C^{\text {Pub }}\right)$ analogously.

\subsubsection{The Bird solution}

The Bird solution is defined as follows. Let $\Pi^{*}(c)$ be the set of orders obtained in Prim's algorithm. For all $\pi \in \Pi^{*}(c)$, let $y_{\pi_{i}}^{b \pi}=\min _{k=0, \ldots, i-1} c_{\pi_{k} \pi_{i}}$ where 
$\pi_{0}=0$. The Bird solution is

$$
y^{b}=\frac{1}{\left|\Pi^{*}(c)\right|} \sum_{\pi \in \Pi^{*}(c)} y^{b \pi} .
$$

Proposition 5 For all $\pi \in \Pi^{*}(c), y^{b \pi}=y^{r \pi}(C)$.

The Bird allocation is thus a very special case of our method, as it only uses the reduced marginal cost vector of $C$ related to permutation $\pi$ if $\pi$ is a permutation corresponding to an order in which we construct the mcst using Prim's algorithm.

We thus obtain an alternative proof of the stability of the Bird allocation. In addition, we can see that the permutations used for the Bird allocation are such that there are no modifications to do on $C$ before computing the marginal cost vector. For other permutations, we usually obtain that the corresponding marginal cost vector (without modifying $C$ ) is not in the core.

Notice that if $\left|\Pi^{*}(c)\right|=1$ the Bird allocation consists of a single extreme allocation of the core. In terms of fairness, this is hard to justify, providing additional ammunition to the criticisms of Dutta and Kar (2004) and Bergantiños and Vidal-Puga (2007).

\subsubsection{The cycle-complete solution}

To define the cycle-complete solution, we need to define the irreducible and cycle-complete cost matrices.

From any cost matrix $c$, we can define the irreducible cost matrix $c^{*}$ as follows:

$$
c_{i j}^{*}=\min _{p_{i j} \in P_{i j}\left(N_{0}\right)} \max _{e \in p_{i j}} c_{e} \text { for all } i, j \in N_{0} .
$$

From any cost matrix $c$, we can define the cycle-complete cost matrix $c^{* *}$ as follows:

$$
\begin{aligned}
c_{i j}^{* *} & =\max _{k \in N \backslash\{i, j\}}\left(c^{N \backslash\{k\}}\right)_{i j}^{*} \text { for all } i, j \in N \\
c_{0 i}^{* *} & =\max _{k \in N \backslash\{i\}}\left(c^{N \backslash\{k\}}\right)_{0 i}^{*} \text { for all } i \in N
\end{aligned}
$$

where $\left(c^{N \backslash\{k\}}\right)^{*}$ indicates the matrix that we first restricted to agents in $N \backslash\{k\}$ before transforming into an irreducible matrix. 
The cycle complete matrix can also be defined using cycles (Trudeau, 2012): for edge $(i, j)$, we look at cycles that go through $i$ and $j$. If there is one such cycle such that its most expensive edge is cheaper than a direct connection on edge $(i, j)$, we assign this cost to edge $(i, j)$.

Let $C^{* *}$ be the characteristic cost function associated with the mest problem $\left(N, c^{* *}\right)$. The cycle-complete solution $y^{c c}$ is the Shapley value of $C^{* *}$.

Let $\Gamma^{e}$ be the set of elementary cost matrices: for any $c \in \Gamma^{e}$ and any $i, j \in N_{0}, c_{i j} \in\{0,1\}$. We show that for elementary cost matrices, the cyclecomplete solution corresponds to the selective value.

Proposition 6 For any elementary cost matrix $c \in \Gamma^{e}, y^{s}(C)=y^{c c}$.

An alternative explanation of the above result is that the changes from the original to the cycle-complete cost matrix are the same as those imposed by our method. If node $i$ has two free distinct paths to node $j$, say with the help of $S$ and $T$, she will obtain the cost savings with both coalitions. This will result in $\widehat{C}(\{i, j\})=0$, the same result as if we modified directly the matrix into a cycle-complete matrix.

\subsubsection{The folk solution}

The folk solution is the Shapley value of the cost game associated with the irreducible cost matrix $c^{*}$ defined in the previous subsection. As for the cyclecomplete solution, we show a link between our method and the folk solution in elementary mcst problems.

Proposition 7 For any elementary cost matrix $c \in \Gamma^{e}, y^{s}\left(C^{P u b}\right)=y^{f}$.

We thus obtain the new result that for elementary mcst problems, the folk solution is the permutation-weighted average of the extreme core allocations of $\operatorname{Core}\left(C^{P u b}\right)$.

Therefore, we obtain, for elementary cost matrices, a clear distinction between the folk and cycle-complete solutions. They both are selective values: the former for the core of the public mcst problem and the latter for the core of the (private) mcst problem.

In Trudeau (2013), it was already noted that the folk solution was axiomatized by properties that fit better with the interpretation of the public game. Our result clearly shows why.

We conclude this section with a computation of the various solutions for our running example. 
Example 5 Consider the mcst problem illustrated in Example 1. The table below shows the shares for the various cost sharing solutions discussed in this section.

$\begin{array}{lllll} & y^{s}(C) & y^{c c} & y^{s}\left(C^{P u b}\right) & y^{f} \\ y_{1} & -1.08 & -1.00 & 0.25 & 1.00 \\ y_{2} & 5.17 & 5.00 & 5.25 & 5.00 \\ y_{3} & 4.46 & 4.50 & 4.25 & 4.00 \\ y_{4} & 3.46 & 3.50 & 2.25 & 2.00\end{array}$

\section{Discussion}

The result of the previous section on the folk and cycle-complete solutions does not hold for non-elementary cost matrices. For those, we can compute the folk and cycle-complete solutions by decomposing the cost matrix into a series of elementary cost matrices and summing up. While that approach is computationally advantageous, one of the disadvantage is that, in general, we have that $\operatorname{Core}\left(C^{* *}\right)$ is a strict subset of $\operatorname{Core}(C)$ and $\operatorname{Core}\left(C^{*}\right)$ is a strict subset of Core $\left(C^{P u b}\right)$ (which is itself also a strict subset of $\operatorname{Core}(C)$ ); the cycle-complete and folk solutions are no longer the permutation-weighted averages of the extreme allocations of, respectively, the core of the private mcst game and the core of the public mest game.

If we are willing to forego the piecewise pinearity property, we can use $y^{s}(C)$ and $y^{s}\left(C^{P u b}\right)$ as non-piecewise linear extensions of, respectively, the cycle-complete and folk solutions.

Given that the reduced game approach allows us to find the full sets of extreme points of the core just as for the assignment problem (Núñez and Rafels, 2003), it is worth exploring if the two problems share more characteristics. In particular, the assignment problem satisfies the CoMa property, meaning that all of the extreme points of its core are marginal vectors (Hamers et al., 2002). This is not true for most problems. An example is provided in appendix.

\section{References}

Bahel, E. and Trudeau, C. (2014). Stable lexicographic rules for shortest path games. Economic Letters, 125:266-269. 
Bergantiños, G. and Vidal-Puga, J. (2007). A fair rule in minimum cost spanning tree problems. Journal of Economic Theory, 137(1):326-352.

Bird, C. (1976). On cost allocation for a spanning tree: a game theoretic approach. Networks, 6:335-350.

Bogomolnaia, A. and Moulin, H. (2010). Sharing the cost of a minimal cost spanning tree: Beyond the folk solution. Games and Economics Behavior, 69:238-248.

Davis, M. and Maschler, M. (1965). The kernel of a cooperative game. Naval Research Logistics Quarterly, 12:223-259.

Driessen, T. (1988). Cooperative games, solutions, and applications. Kluwer Academic Publisher.

Dutta, B. and Kar, A. (2004). Cost monotonicity, consistency and minimum cost spanning tree games. Games and Economic Behavior, 48(2):223-248.

Feltkamp, V., Tijs, S., and Muto, S. (1994). On the irreducible core and the equal remaining obligations rule of minimum cost spanning extension problems. Technical Report 106, CentER DP 1994 nr.106, Tilburg University, The Netherlands.

Funaki, Y., Tijs, S., and Brânzei, R. (2007). Leximals, the lexicore and the average lexicographic value. CentER Discussion Paper 2007-97, Tilburg University.

Granot, D. and Huberman, G. (1981). On minimum cost spanning tree games. Mathematical Programming, 21:1-18.

Granot, D. and Huberman, G. (1984). On the core and nucleolus of minimum cost spanning tree problems. Mathematical Programming, 29:323-347.

Hamers, H., Klijn, F., Solymosi, T., Tijs, S., and Pere Villar, J. (2002). Assignment games satisfy the CoMa-property. Games and Economic Behavior, 38(2):231-239.

Hwang, F. and Richards, D. S. (1992). Steiner tree problems. Networks, 22(1):55-89. 
Kongo, T., Funaki, Y., Branzei, R., and Tijs, S. (2010). Non-cooperative and axiomatic characterizations of the average lexicographic value. International Game Theory Review, 12(4):417-435.

Núñez, M. and Rafels, C. (1998). On extreme points of the core and reduced games. Annals of Operations Research, 84:121-133.

Núñez, M. and Rafels, C. (2003). Characterization of the extreme core allocations of the assignment game. Games and Economic Behavior, 44(2):311331.

Pérez-Castrillo, D. and Sotomayor, M. (2002). A simple selling and buying procedure. Journal of Economic Theory, 103:461-474.

Potters, J., Poos, R., Tijs, S., and Muto, S. (1989). Clan games. Games and Economic Behavior, 1:275-293.

Skorin-Kapov, D. (1995). On the core of the minimum cost steiner tree game in networks. Annals of Operations Research, 57:233-249.

Tijs, S., Borm, P., Lohmann, E., and Quant, M. (2011). An average lexicographic value for cooperative games. European Journal of Operational Research, 213(1):210-220.

Tijs, S. H. (2005). The first steps with Alexia, the Average lexicographic value. CentER DP 2005-12, Tilburg University.

Trudeau, C. (2012). A new stable and more responsible cost sharing solution for mest problems. Games and Economic Behavior, 75(1):402-412.

Trudeau, C. (2013). Characterizations of the Kar and folk solutions for minimum cost spanning tree problems. International Game Theory Review, $15(2)$.

Vidal-Puga, J. (2004). Bargaining with commitments. International Journal of Game Theory, 33(1):129-144. 


\section{A Appendix - Proofs}

\section{A.1 Proposition 1}

Let $S \subseteq N \backslash P^{\pi i}$ and let $t^{S}$ be a tree that connects all the nodes in $S$ to the source using a set $T^{S} \subseteq P^{\pi i}$ of priced nodes. We need to prove

$$
y^{r \pi}(S) \leq \sum_{e \in t^{S}} c_{e}-y^{r \pi}\left(T^{S}\right)
$$

or, equivalently,

$$
y^{r \pi}\left(S \cup T^{S}\right) \leq \sum_{e \in t^{S}} c_{e}
$$

Assume there exists $t^{*} \in \mathcal{T}^{*}(c)$ that is also an optimal tree in $\left(N, N, y^{r \pi}, c\right)$. Hence

$$
\sum_{e \in t^{*}} c_{e}-y^{r \pi}(N) \leq \sum_{e \in t^{S}} c_{e}-y^{r \pi}\left(S \cup T^{S}\right) .
$$

Since $\sum_{e \in t^{*}} c_{e}=y^{r \pi}(N)$, we have that (1) and (2) coincide.

We still need to prove that there exists $t^{*} \in \mathcal{T}^{*}(c)$ that is also an optimal tree in $\left(N, N, y^{r \pi}, c\right)$. We will prove a stronger result: that any $t^{*} \in \mathcal{T}^{*}(c)$ is an optimal tree in any $\left(N, P^{\pi i}, y^{r \pi}, c\right)$. Assume w.l.o.g. $\pi=[1 \ldots n]$. For notational convenience, let $P^{\pi n+1}=\emptyset$. We proceed by backwards induction on $i$. For $i=n+1$, the result holds trivially. Assume the result holds for $i+1 \leq n+1$. Let $t^{*} \in \mathcal{T}^{*}(c)$. We have to prove that $t^{*}$ is an optimal tree on $\left(N, P^{\pi i}, y^{r \pi}, c\right)$, i.e. for each tree $t$ that connects all the nodes in $\left(N \backslash P^{\pi i}\right) \cup T$ to the source, with $T \subseteq P^{\pi i}$, it holds that

$$
\sum_{e \in t^{*}} c_{e}-y^{r \pi}\left(P^{\pi i}\right) \leq \sum_{e \in t} c_{e}-y^{r \pi}(T)
$$

By induction hypothesis, $t^{*}$ is an optimal tree in $\left(N, P^{\pi i+1}, y^{r \pi}, c\right)$. Assume first $i \in T$. Then, $\sum_{e \in t^{*}} c_{e}-y^{r \pi}\left(P^{\pi i+1}\right) \leq \sum_{e \in t} c_{e}-y^{r \pi}(T \backslash i)$, which is equivalent to (3). Assume now $i \notin T$. Hence

$$
\begin{aligned}
\sum_{e \in t^{*}} c_{e}-y^{r \pi}\left(P^{\pi i}\right) & =\sum_{e \in t^{*}} c_{e}-y^{r \pi}\left(P^{\pi i+1}\right)-y_{i}^{r \pi} \\
& =C\left(N, P^{\pi i+1}, y^{r \pi}, c\right)-y_{i}^{r \pi} \\
& =C\left(N \backslash P^{\pi i+1}, P^{\pi i+1}, y^{r \pi}, c\right)-y_{i}^{r \pi} .
\end{aligned}
$$


By definition, $y_{i}^{r \pi}=C\left(N \backslash P^{\pi i+1}, P^{\pi i+1}, y^{r \pi}, c\right)-C\left(N \backslash P^{\pi i}, P^{\pi i+1}, y^{r \pi}, c\right)$. Hence the above expression equals $C\left(N \backslash P^{\pi i}, P^{\pi i-1}, y^{r \pi}, c\right)$ which, by definition, is less or equal than $\sum_{e \in t} c_{e}-y^{r \pi}(T)$.

\section{A.2 Lemma 1}

Given that $\widehat{C}(S) \leq C(S)$ for all $S \subset N$ and $\widehat{C}(N)=C(N)$, it is obvious that Core $(\widehat{C}) \subseteq \operatorname{Core}(C)$. We show that $\operatorname{Core}(C) \subseteq$ Core $(\widehat{C})$. Suppose, on the contrary, that there exists $y \in \operatorname{Core}(C)$ such that $y \notin$ Core $(\widehat{C})$. Then, there exists $\emptyset \neq S \subset N$ such that $\widehat{C}(S)<y(S) \leq C(S)$. Stated otherwise, there exists $\emptyset \neq T \subset N \backslash S$ such that

$$
\widehat{C}(S)=\widehat{C}(S \cup T)+\widehat{C}(N \backslash T)-\widehat{C}(N)<y(S) \leq C(S) .
$$

Suppose first that $|S|=n-2$. Then,

$$
\widehat{C}(S)=C(S \cup T)+C(N \backslash T)-C(N)<y(S) \leq C(S)
$$

as $|S \cup T|,|N \backslash T| \geq n-1$. Since $y \in \operatorname{Core}(C)$, we must have that $y(S)+$ $y(T)+y(N \backslash(S \cup T))=C(N)$. We have the following core conditions:

$$
\begin{aligned}
y(S)+y(T) & \leq C(S \cup T) \\
y(S)+y(N \backslash(S \cup T)) & \leq C(N \backslash T) .
\end{aligned}
$$

Adding these two constraints, we obtain

$$
\begin{aligned}
2 y(S)+y(T)+y(N \backslash(S \cup T)) & \leq C(S \cup T)+C(N \backslash T) \\
y(S) & \leq C(S \cup T)+C(N \backslash T)-C(N)=\widehat{C}(S) .
\end{aligned}
$$

Therefore, we have a contradiction with (4).

We have thus shown that for $y \in \operatorname{Core}(C)$, we need $y(S) \leq \widehat{C}(S)$ if $|S|=$ $n-2$. We can then use a recursive argument to show that if $y \in \operatorname{Core}(C)$ implies that $y(S) \leq \widehat{C}(S)$ for all $S$ such that $|S| \geq n-k$, it also implies that $y(S) \leq \widehat{C}(S)$ for all $S$ such that $|S|=n-k-1$. Thus, $y(S) \leq \widehat{C}(S)$ for all $S \subseteq N$ is a necessary condition for $y \in \operatorname{Core}(C)$ and $\operatorname{Core}(C) \subseteq \operatorname{Core}(\widehat{C})$. 


\section{A.3 Lemma 2}

Let $t^{1}$ be an optimal tree in $\left(N \backslash\left\{p_{1}\right\}, P_{1} \backslash\left\{p_{1}\right\}, y^{r \pi}, c\right)$. We will prove that $t^{1}$ is also an optimal tree in both $\left(N \backslash\left\{p_{1}\right\}, P^{a} \backslash\left\{p_{1}\right\}, y^{r \pi}, c\right)$ and $\left(N \backslash\left\{p_{1}\right\}, P^{b} \backslash\left\{p_{1}\right\}, y^{r \pi}, c\right)$. Notice first that $t^{1}$ connects to the source all the nodes in $N \backslash P_{1}$, which includes both $S^{a}=N \backslash P^{a}$ and $S^{b}=N \backslash P^{b}$. Let $t^{a}$ be an optimal tree in $\left(N \backslash\left\{p_{1}\right\}, P^{a} \backslash\left\{p_{1}\right\}, y^{r \pi}, c\right)$ that connects all the nodes in $S^{a}$ to the source (the case for $\left(N \backslash\left\{p_{1}\right\}, P^{b} \backslash\left\{p_{1}\right\}, y^{r \pi}, c\right)$ is analogous). In case there are more than one possible optimal tree, we choose $t^{a}$ with maximal number of nodes $\left|t^{a}(N)\right|$. We need to prove that $c\left(t^{1}\right)-y^{r \pi}\left(t^{1}(N) \cap P^{a}\right) \leq$ $c\left(t^{a}\right)-y^{r \pi}\left(t^{a}(N) \cap P^{a}\right)$. Assume first $P^{a} \backslash P_{1} \subseteq t^{a}(N)$. Since $t^{1}$ is optimal in $\left(N \backslash\left\{p_{1}\right\}, P_{1} \backslash\left\{p_{1}\right\}, y^{r \pi}, c\right)$, we have

$$
\begin{aligned}
c\left(t^{1}\right)-y^{r \pi}\left(t^{1}(N) \cap P^{a}\right) & =c\left(t^{1}\right)-y^{r \pi}\left(t^{1}(N) \cap P_{1}\right)-y^{r \pi}\left(P^{a} \backslash P_{1}\right) \\
& \leq c\left(t^{a}\right)-y^{r \pi}\left(t^{a}(N) \cap P_{1}\right)-y^{r \pi}\left(P^{a} \backslash P_{1}\right) \\
& =c\left(t^{a}\right)-y^{r \pi}\left(t^{a}(N) \cap P^{a}\right) .
\end{aligned}
$$

Assume now there exists $p_{2} \in P^{a} \backslash P_{1}$ such that $p_{2} \notin t^{a}(N)$. Assume w.l.o.g. that $p_{2}^{*} \in t^{a}(N)_{0}$, i.e. the immediate predecessor of $p_{2}$ in $t^{*}$ is connected to the source (or it is the source itself) through $t^{a}$. Let $P_{2}$ be the maximal subset of $P^{a}$ whose nodes are connected to $p_{2}$ in $t^{*}$ (including $p_{2}$ itself) without using any node in $t^{a}(N)$ (this implies $p_{2} \in P_{2}$ and $p_{2}^{*} \notin P_{2}$ ). Let $R^{*}=\left\{i \in N_{0} \backslash P_{2}: i^{*} \in P_{2}\right\} \cup\left\{p_{2}^{*}\right\}$ be the set of nodes in $N_{0}$ (and also in $\left.t^{a}(N)_{0}\right)$ that are linked in $t^{*}$ to some node in $P_{2}$. Assume w.l.o.g. $R^{*}=$ $\left\{1, \ldots, k-1, p_{2}^{*}\right\}$. For each $i \in R^{*} \backslash\left\{p_{2}^{*}\right\}$, let $F^{* i}$ be the set of nodes that follow node $i$ in $t^{*}$ (including node $i$ itself). We define the set of edges $E=\left\{e_{1}, \ldots, e_{k-1}\right\} \subset N_{0}^{p}$ inductively as follows: Let $e_{1}=\left(i_{1}, i_{1}^{\prime}\right) \in N_{0}^{p}$ be the first edge in the (unique) path in $t^{a}$ from node 1 to node $p_{2}^{*}$ such that $i_{1} \in F^{* 1}$ and $i_{1}^{\prime} \notin F^{* 1}$. Let $e_{2}=\left(i_{2}, i_{2}^{\prime}\right) \in N_{0}^{p}$ be the first edge in the (unique) path in $t^{a}$ from node 2 to node $p_{2}^{*}$ such that $i_{2} \in F^{* 1} \cup F^{* 2}$ and $i_{2}^{\prime} \notin F^{* 1} \cup F^{* 2}$. Let $e_{3}=\left(i_{3}, i_{3}^{\prime}\right) \in N_{0}^{p}$ be the first edge in the (unique) path in $t^{a}$ from node 3 to node $p_{2}^{*}$ such that $i_{3} \in F^{* 1} \cup F^{* 2} \cup F^{* 3}$ and $i_{3}^{\prime} \notin F^{* 1} \cup F^{* 2} \cup F^{* 3}$. And so on. Thus, $E$ is the set of edges that connect $R^{*}$ in $t^{a}$, and so that $t^{a} \backslash E$ leaves $k$ connected components. Since nodes in $R^{*}$ are connected in $t^{*}$ using the $\operatorname{arcs}$ in $E^{*}=\left\{(i, j) \in t^{*}: i, j \in R^{*} \cup P_{2}\right\}$, we deduce that

$$
t^{2}=\left(t^{a} \backslash E\right) \cup E^{*}
$$


is, like $t^{a}$, a tree that connects all the nodes in $N \backslash P^{a}$ to the source and, moreover,

$$
t^{a}(N)=t^{2}(N) \backslash P_{2}
$$

Optimality of $t^{*}$ in $\left(N, N, y^{r \pi}, c\right)$ implies that

$$
c\left(E^{*}\right)-y^{r \pi}\left(P_{2}\right) \leq c(E)
$$

because, otherwise, $\left(t^{*} \backslash E^{*}\right) \cup E$ would improve $t^{*}$. We now check that $t^{2}$ is also, like $t^{a}$, an optimal tree in $\left(N \backslash\left\{p_{1}\right\}, P^{a} \backslash\left\{p_{1}\right\}, y^{r \pi}, c\right)$ :

$$
\begin{aligned}
c\left(t^{2}\right)-y^{r \pi}\left(t^{2}(N) \cap P^{a}\right) & \stackrel{(5)}{=} c\left(t^{a}\right)-c(E)+c\left(E^{*}\right)-y^{r \pi}\left(t^{2}(N) \cap P^{a}\right) \\
& \stackrel{(7)}{\leq} c\left(t^{a}\right)+y^{r \pi}\left(P_{2}\right)-y^{r \pi}\left(t^{2}(N) \cap P^{a}\right) \\
& \stackrel{(6)}{=} c\left(t^{a}\right)-y^{r \pi}\left(t^{a}(N) \cap P^{a}\right)
\end{aligned}
$$

which contradicts $\left|t^{a}(N)\right|$ be maximal.

\section{A.4 Proposition 2}

Fix $t^{*}=\left\{\left(i, i^{*}\right)\right\}_{i \in N} \in \mathcal{T}^{*}(c)$. Given $S \subseteq N$ and $P=N \backslash S$, let $\pi \in \Pi$ be an order $t^{*}$-compatible with $P$. We prove that the following two statements hold:

(I) Either $y^{r \pi}(S)=C(S)$ or there exists $\emptyset \neq T^{\prime} \subset P$ such that

(I a) $y^{r \pi^{a}}\left(T^{\prime}\right)=y^{r \pi}\left(T^{\prime}\right)$ for all $\pi^{a} \in \Pi$ order $t^{*}$-compatible with $T^{\prime}$, and

(Ib) $y^{r \pi^{b}}\left(P \backslash T^{\prime}\right)=y^{r \pi}\left(P \backslash T^{\prime}\right)$ for all $\pi^{b} \in \Pi$ order $t^{*}$-compatible with $P \backslash T^{\prime}$.

(II) $y^{r \pi}(S)=\widehat{C}(S)$.

We proceed by induction on $|P|$. For $P=\emptyset$, both statements hold trivially. Assume now both statements hold when $|P|<\alpha$ and suppose $|P|=\alpha$ for some $\alpha>0$.

We first prove statement (I). Let $p_{1}=\pi_{s} \in P$ be the first element in $P$ according to $\pi$ (that is, $i<s$ implies $\pi_{i} \notin P$ ). Hence, for all $\pi_{i} \in P \backslash\left\{p_{1}\right\}$, we have $s<i$. Under internal $t^{*}$-compatibility, we deduce $p_{1} \notin F^{* i}$ for all $i \in P \backslash\left\{p_{1}\right\}$. 
Let $S^{\prime}=S \cup\left\{p_{1}\right\}$ and $P^{\prime}=P \backslash\left\{p_{1}\right\}$. Let $P_{1}=\left\{i \in N: \tau_{i p_{1}}^{*} \subseteq P\right\}$, where $\tau_{i p_{1}}^{*}$ is the (unique) path from node $i$ to node $p_{1}$ in $t^{*}$, be the set of nodes in $P$ that are adjacent to $p_{1}$.

Let $t^{\prime}$ be an optimal tree in $\left(N \backslash\left\{p_{1}\right\}, P^{\prime}, y^{r \pi}, c\right)$. That is:

$$
\begin{gathered}
S \subseteq t^{\prime}(N) \\
p_{1} \notin t^{\prime}(N) \\
c\left(t^{\prime}\right)-y^{r \pi}\left(t^{\prime}(N) \cap P^{\prime}\right)=\min _{T \subseteq P^{\prime}}\left\{C(S \cup T)-y^{r \pi}(T)\right\} .
\end{gathered}
$$

In case there are more than one tree satisfying the above conditions, we take $t^{\prime}$ such that $\left|t^{\prime}(N)\right|$ is maximal among them.

Assume first $S=t^{\prime}(N)$, which means $t^{\prime}(N) \cap P^{\prime}=\emptyset$ and $c\left(t^{\prime}\right)=C(S)$. Moreover, under Lemma 2, we deduce that $P=P_{1}$. We will prove that $y^{r \pi}(S)=C(S)$, so that statement (I) holds.

Let $\sigma \in \Pi$ defined from $\pi$ by moving nodes in $P$ to the last positions, leaving the rest of the order unaffected ${ }^{3}$. Hence, $P=P^{\sigma s^{\prime}}$ for some $s^{\prime} \geq$ $s$. From the proof of Proposition 1, we know that $y_{N \backslash P^{\sigma s^{\prime}}}^{r \sigma}$ belongs to the core of $\left(N, P^{\sigma s^{\prime}}, y^{r \sigma}, c\right)$. Since $P=P^{\sigma s^{\prime}}$, this can be rewritten as $y_{S}^{r \sigma} \in$ Core $\left(N, P, y^{r \sigma}, c\right)$, which implies $y^{r \sigma}(S)=C\left(S, P, y^{r \sigma}, c\right)$. Moreover, we know that $C\left(S, P, y^{r \pi}, c\right)=C(S)$. Hence, it is enough to prove that $y^{r \sigma}=$ $y^{r \pi}$. Let $\pi_{m} \in S$ such that $\left\{\pi_{s}, \ldots, \pi_{m-1}\right\} \subseteq P=P_{1}$. We will prove that $y^{r \pi}=y^{r \pi^{\prime}}$ where $\pi^{\prime} \in \Pi$ coincides with $\pi$ after changing nodes $\pi_{m-1}$ and $\pi_{m}$, i.e.

$$
\pi^{\prime}=\left[\pi_{1}, \ldots, \pi_{m-2}, \pi_{m}, \pi_{m-1}, \pi_{m+1}, \ldots, \pi_{n}\right]
$$

so that a repeated reasoning leaves to $y^{r \pi}=y^{r \sigma}$.

It is clear that $y_{\pi_{i}}^{r \pi}=y_{\pi_{i}}^{r \pi^{\prime}}$ for all $i>m$. We now check that $y_{\pi_{m}}^{r \pi}=y_{\pi_{m}}^{r \pi^{\prime}}$. Under $t^{*}$-compatibility, it is straightforward to check that $P_{1}$ is a maximal connected component of both $\left\{\pi_{m+1}, \ldots, \pi_{n}\right\}$ and $\left\{\pi_{m-1}, \pi_{m+1}, \ldots, \pi_{n}\right\}$. Under Lemma 2, there exists an optimal tree $t^{s}$ in both

$$
\left(\left\{\pi_{1}, \ldots, \pi_{m-1}\right\},\left\{\pi_{m+1}, \ldots, \pi_{n}\right\}, y^{r \pi}, c\right)
$$

and

$$
\left(\left\{\pi_{1}, \ldots, \pi_{m-2}\right\},\left\{\pi_{m-1}, \pi_{m+1}, \ldots, \pi_{n}\right\}, y^{r \pi}, c\right) .
$$

\footnotetext{
${ }^{3}$ For example, if $\pi=[123456789]$ and $P=\{3,4,6,8\}$, then $\sigma^{P}=[125793468]$.
} 
Hence,

$$
\begin{aligned}
y_{\pi_{m}}^{r \pi}= & C\left(\left\{\pi_{1}, \ldots, \pi_{m}\right\},\left\{\pi_{m+1}, \ldots, \pi_{n}\right\}, y^{r \pi}, c\right) \\
& -C\left(\left\{\pi_{1}, \ldots, \pi_{m-1}\right\},\left\{\pi_{m+1}, \ldots, \pi_{n}\right\}, y^{r \pi}, c\right) \\
= & c\left(t^{*}\right)-y^{r \pi}\left(\left\{\pi_{m+1}, \ldots, \pi_{n}\right\}\right) \\
& -c\left(t^{s}\right)+y^{r \pi}\left(t^{s}(N) \cap\left\{\pi_{t+1}, \ldots, \pi_{n}\right\}\right)
\end{aligned}
$$

and

$$
\begin{aligned}
y_{\pi_{m}}^{r \pi^{\prime}}= & C\left(\left\{\pi_{1}, \ldots, \pi_{m-2}, \pi_{m}\right\},\left\{\pi_{m-1}, \pi_{m+1}, \ldots, \pi_{n}\right\}, y^{r \pi}, c\right) \\
& -C\left(\left\{\pi_{1}, \ldots, \pi_{m-2}\right\},\left\{\pi_{m-1}, \pi_{m+1}, \ldots, \pi_{n}\right\}, y^{r \pi}, c\right) \\
= & c\left(t^{*}\right)-y^{r \pi}\left(\left\{\pi_{m-1}, \pi_{m+1}, \ldots, \pi_{n}\right\}\right) \\
& -c\left(t^{s}\right)+y^{r \pi}\left(t^{s}(N) \cap\left\{\pi_{m-1}, \pi_{m+1}, \ldots, \pi_{n}\right\}\right) .
\end{aligned}
$$

Since $\pi_{m-1} \in P_{1}$, we deduce that $\pi_{m-1}$ does not belong to the same connected component as $\pi_{m}$, and hence $\pi_{m-1} \in t^{s}(N)$. This implies that (8) and (9) coincide and thus $y_{\pi_{m}}^{r \pi}=y_{\pi_{m}}^{r \pi^{\prime}}$. An analogous reasoning leads to $y_{\pi_{m-1}}^{r \pi}=y_{\pi_{m-1}}^{r \pi^{\prime}}$ and from it, $y_{\pi_{i}}^{r \pi}=y_{\pi_{i}}^{r \pi^{\prime}}$ for all $i<m-1$, so that $y^{r \pi}=y^{r \pi^{\prime}}$, as desired.

Assume now $S \subset t^{\prime}(N)$. Let $T^{\prime}=t^{\prime}(N) \backslash S$. It is straightforward to check that $\emptyset \neq T^{\prime} \subset P$. We will prove that $(\mathrm{I} a)$ and $(\mathrm{I} b)$ hold with this $T^{\prime}$. As a previous step, we need to prove the following Claim:

Claim A: For all $i \in N \backslash\left\{p_{1}\right\}, i^{*} \in S \cup T^{\prime} \Rightarrow i \in S \cup T^{\prime}$.

Proof. Assume, on the contrary, that there exists some $i \in N \backslash\left\{p_{1}\right\}$ such that $i^{*} \in S \cup T^{\prime}$ and $i \notin S \cup T^{\prime}$. That is, $i^{*} \in t^{\prime}(N)$ and $i \notin t^{\prime}(N)$.

Let $Q=\left\{j \in N \backslash T^{\prime}: \tau_{i j}^{*} \subseteq N \backslash T^{\prime}\right\}$, where $\tau_{i j}^{*}$ is the (unique) path form node $i$ to node $j$ in $t^{*}$. Thus, $Q$ is the set of followers of node $i$ without leaving $P \backslash T^{\prime}$. Hence, $Q \subseteq P \backslash T^{\prime}$. Since $p_{1} \notin F^{* j}$ for all $j \in P^{\prime}$, we deduce that $p_{1} \notin Q$. Let $R=\left\{j \in N: j^{*} \in Q\right\}$ be the set of nodes in $S$ that immediately follow some node in $Q$ (case $R=\emptyset$ is also possible). Denote $R=\left\{r_{1}, \ldots, r_{k}\right\}$. We define the set of edges $E=\left\{e_{1}, \ldots, e_{k}\right\} \subset N^{p}$ inductively as follows: Let $e_{1}=\left(i_{1}, i_{1}^{\prime}\right) \in N^{p}$ be the first edge in the (unique) path in $t^{\prime}$ from $r_{1}$ to the source such that $i_{1} \in F^{* r_{1}}$ and $i_{1}^{\prime} \notin F^{* r_{1}}$. Let $e_{2}=\left(i_{2}, i_{2}^{\prime}\right) \in N^{p}$ be the first edge in the (unique) path in $t^{\prime}$ from $r_{2}$ to the source such that $i_{2} \in F^{* r_{1}} \cup F^{* r_{2}}$ and $i_{2}^{\prime} \notin F^{* r_{1}} \cup F^{* r_{2}}$. Let $e_{3}=\left(i_{3}, i_{3}^{\prime}\right) \in N^{p}$ be the first edge in the (unique) path in $t^{\prime}$ from $r_{3}$ to the source such that 
$i_{3} \in F^{* r_{1}} \cup F^{* r_{2}} \cup F^{* r_{3}}$ and $i_{3}^{\prime} \notin F^{* r_{1}} \cup F^{* r_{2}} \cup F^{* r_{3}}$. And so on. Thus, $E$ is the set of edges that connect $R$ to the source in $t^{\prime}$. Now,

$$
t^{1}=\left(t^{*} \backslash\left\{\left(j, j^{*}\right)\right\}_{j \in Q \cup R}\right) \cup E
$$

is a tree that connects all the nodes in $S$ to the source using nodes in $P \backslash Q$. Since $t^{*}$ is optimal in $\left(S, P, y^{r \pi}, c\right)$, we deduce that

$$
c\left(t^{*}\right)-y^{r \pi}(P) \leq c\left(t^{1}\right)-y^{r \pi}(P \backslash Q)
$$

or, equivalently,

$$
c\left(t^{*}\right) \leq c\left(t^{1}\right)+y^{r \pi}(Q)
$$

Now,

$$
t^{2}=\left(t^{\prime} \backslash E\right) \cup\left\{\left(j, j^{*}\right)\right\}_{j \in Q \cup R}
$$

is a tree that connects all the nodes in $S$ to the source using nodes in $T^{\prime} \cup Q \subseteq$ $P^{\prime}$. Since $t^{\prime}$ is optimal in $\left(S, P^{\prime}, y^{r \pi}, c\right)$, we deduce that

$$
c\left(t^{\prime}\right)-y^{r \pi}\left(T^{\prime}\right) \leq c\left(t^{2}\right)-y^{r \pi}\left(T^{\prime} \cup Q\right)
$$

or, equivalently,

$$
c\left(t^{\prime}\right) \leq c\left(t^{2}\right)-y^{r \pi}(Q) .
$$

By applying (11) and (13),

$$
\begin{aligned}
c\left(t^{2}\right) & \stackrel{(13)}{\geq} c\left(t^{\prime}\right)+y^{r \pi}(Q) \stackrel{(11)}{\geq} c\left(t^{\prime}\right)+c\left(t^{*}\right)-c\left(t^{1}\right) \\
& \stackrel{(10)}{=} c\left(t^{\prime}\right)+c\left(t^{*}\right)-c\left(t^{*}\right)+c\left(\left\{\left(j, j^{*}\right)\right\}_{j \in Q \cup R}\right)-c(E) \\
& =c\left(t^{\prime}\right)+c\left(\left\{\left(j, j^{*}\right)\right\}_{j \in Q \cup R}\right)-c(E) \\
& \stackrel{(12)}{=} c\left(t^{2}\right) .
\end{aligned}
$$

This implies $c\left(t^{2}\right)=c\left(t^{\prime}\right)+y^{r \pi}(Q)$, so

$$
c\left(t^{2}\right)-y^{r \pi}\left(T^{\prime} \cup Q\right)=c\left(t^{\prime}\right)-y^{r \pi}\left(T^{\prime}\right)
$$

and hence $t^{2}$ is also an optimal tree in $\left(S, P^{\prime}, y^{r \pi}, c\right)$ with $\left|t^{2}(N)\right|=\left|t^{\prime}(N)\right|+$ $|Q|$, which contradicts that $\left|t^{\prime}(N)\right|$ is maximum among these optimal trees (notice that $i \in Q \neq \emptyset$ ). This contradiction completes the proof of Claim A. 
We can now prove that ( $\mathrm{I} a$ ) holds with $T^{\prime}$. Under the induction hypothesis on statement (II), we have $y^{r \pi^{a}}\left(N \backslash T^{\prime}\right)=\widehat{C}\left(N \backslash T^{\prime}\right)$ for all $\pi^{a} \in \Pi$ order $t^{*}$ compatible with $T^{\prime}$. On the other hand, if $\pi$ is $t^{*}$-compatible with $T^{\prime}$, then we can also apply the induction hypothesis on (II) to deduce that $y^{r \pi}\left(N \backslash T^{\prime}\right)=$ $\widehat{C}\left(N \backslash T^{\prime}\right)$. Hence, it is enough to prove that $\pi$ is indeed $t^{*}$-compatible with $T^{\prime}$. We check both external and internal $t^{*}$-compatibility.

External $t^{*}$-compatibility: Let $i, j$ such that $\pi_{i} \preceq_{*} \pi_{j}$ or $\pi_{i}^{*}=\pi_{j}, \pi_{i} \in T^{\prime}$ and $\pi_{j} \in N \backslash T^{\prime}$. We have three cases: If $\pi_{j} \in S$, then $j<i$ because $\pi$ is externally $t^{*}$-compatible with $P$. If $\pi_{j}=p_{1}$ and $\pi_{i} \preceq_{*} p_{1}$, then $s<i$ because $\pi_{i} \in T^{\prime} \subset P$ and $p_{1}=\pi_{s}$ is the first element of $P$ in $\pi$; by internal $t^{*}$-compatibility with $P$, we deduce $\pi_{i} \npreceq_{*} \pi_{s}=\pi_{j}$, which is a contradiction. If $\pi_{j}=p_{1}$ and $\pi_{i}^{*}=\pi_{j}$, then $j<i$ is equivalent to $s<i$, which holds because $\pi_{i} \in T^{\prime} \subset S$. Finally, if $\pi_{j} \in P \backslash\left(T^{\prime} \cup\left\{p_{1}\right\}\right)$, then $\pi_{j} \notin S \cup T^{\prime} \cup\left\{p_{1}\right\}$; under Claim A, we have $\pi_{j}^{*} \notin S \cup T^{\prime}$; by applying Claim A iteratively, and since $\pi_{i} \preceq_{*} \pi_{j}$, we conclude that $\pi_{i} \notin S \cup T^{\prime}$, which is a contradiction because $\pi_{i} \in T^{\prime}$.

Internal $t^{*}$-compatibility: Let $i, j$ such that $\pi_{i} \preceq_{*} \pi_{j}$ and $\pi_{i}, \pi_{j} \in T^{\prime}$. Since $T^{\prime} \subset P$, we have $\pi_{i}, \pi_{j} \in P$. Hence, $i \leq j$ because $\pi$ is internally $t^{*}$-compatible with $P$.

We now prove that $(\mathrm{I} b)$ holds with $T^{\prime}$. By definition of $y_{p_{1}}^{r \pi}$, both $t^{*}$ and $t^{\prime}$ are optimal trees in $\left(N, P, y^{r \pi}\right)$. Hence, $C(N)-y^{r \pi}(P)=C\left(S \cup T^{\prime}\right)-y^{r \pi}\left(T^{\prime}\right)$. Equivalently,

$$
y^{r \pi}\left(P \backslash T^{\prime}\right)=C(N)-C\left(S \cup T^{\prime}\right) .
$$

Let $\pi^{b} \in \Pi$ be an order $t^{*}$-compatible with $P \backslash T^{\prime}$. We check that $y^{r \pi^{b}}\left(P \backslash T^{\prime}\right)=$ $C(N)-C\left(S \cup T^{\prime}\right)$. By the induction hypothesis on statement (II), we can assume that nodes in $P \backslash T^{\prime}$-follow the same order as in $\pi$. In particular, $p_{1}$ is still the first node in $P \backslash T^{\prime}$ under $\pi^{b}$. Denote $P \backslash T^{\prime}=\left\{p_{1}, \ldots, p_{L}\right\}$ following order $\pi^{b}$, i.e. when $p_{i}=\pi_{i^{b}}^{b}$ and $p_{j}=\pi_{j^{b}}^{b}$, then $i \leq j \Leftrightarrow i^{b} \leq j^{b}$. Internal $t^{*}$-compatibility assures that $p_{i} \preceq_{*} p_{j} \Rightarrow i \leq j$. For each $l \in\{1, \ldots, L\}$, let $G^{l}=\left\{i \in S \cup T^{\prime}: i^{*}=p_{l}\right\}$ be the set of nodes in $S \cup T^{\prime}$ that immediately follow $p_{l}$ in $t^{*}$ (case $G^{l}=\emptyset$ is also possible), and denote $G^{l}=\left\{g_{1}^{l}, \ldots, g_{k_{l}}^{l}\right\}$. We define the set of edges $E^{l}=\left\{e_{1}^{l}, \ldots, e_{k_{l}}^{l}\right\} \subset N^{p}$ inductively as follows: Let $e_{1}^{l}=\left(i_{l 1}, i_{l 1}^{\prime}\right) \in N^{p}$ be the first edge in the (unique) path in $t^{\prime}$ from $g_{1}^{l}$ to the source such that $i_{l 1} \in F^{* g_{1}^{l}}$ and $i_{l 1}^{\prime} \notin F^{* g_{1}^{l}}$. Let $e_{2}^{l}=\left(i_{l 2}, i_{l 2}^{\prime}\right) \in N^{p}$ be the first edge in the (unique) path in $t^{\prime}$ from $g_{2}^{l}$ to the source such that $i_{l 2} \in F^{* g_{1}^{l}} \cup F^{* g_{2}^{l}}$ and $i_{l 2}^{\prime} \notin F^{* g_{1}^{l}} \cup F^{* g_{2}^{l}}$. And so on. Thus, $E^{l}$ is the set of nodes that connect each $G^{l}$ to the source in $t^{\prime}$. We now define the trees 
$t^{0}, t^{1}, \ldots, t^{L}$ inductively as $t^{0}=t^{\prime}$ and

$$
t^{l}=\left(t^{l-1} \cup\left\{\left(i, i^{\prime}\right)\right\}_{i \in G^{l} \cup\left\{p_{l}\right\}}\right) \backslash E^{l}
$$

for each $l=1, \ldots, L$. By optimality of $t^{*}$ and $t^{\prime}$, each $t^{l}$ is also optimal in $\left(N,\left\{p_{l+1}, \ldots, p_{L}\right\}, y^{r \pi}, c\right)$. Hence, we have

$$
\begin{aligned}
y_{p_{L}}^{r \pi} & =c\left(t^{L}\right)-c\left(t^{L-1}\right)=C(N)-C\left(N \backslash\left\{p_{L}\right\}\right) \\
y_{p_{L-1}}^{r \pi} & =c\left(t^{L-1}\right)-c\left(t^{L-2}\right)=C\left(N \backslash\left\{p_{L}\right\}\right)-C\left(N \backslash\left\{p_{L}, p_{L-1}\right\}\right) \\
\quad & \\
y_{p_{1}}^{r \pi} & =c\left(t^{1}\right)-c\left(t^{0}\right)=C\left(S \cup T^{\prime} \cup\left\{p_{1}\right\}\right)-C\left(S \cup T^{\prime}\right)
\end{aligned}
$$

from where we deduce

$$
\begin{aligned}
y_{p_{L}}^{r \pi^{b}} & =C(N)-C\left(N \backslash\left\{p_{L}\right\}\right) \\
y_{p_{L-1}}^{r \pi^{b}} & =C\left(N \backslash\left\{p_{L}\right\}\right)-C\left(N \backslash\left\{p_{L}, p_{L-1}\right\}\right) \\
& \vdots \\
y_{p_{1}}^{r \pi^{b}} & =C\left(S \cup T^{\prime} \cup\left\{p_{1}\right\}\right)-C\left(S \cup T^{\prime}\right)
\end{aligned}
$$

and, adding up these terms, we get

$$
y^{r \pi^{b}}\left(P \backslash T^{\prime}\right)=C(N)-C\left(S \cup T^{\prime}\right)
$$

so that (Ib) comes from (14) and (15).

We now prove statement (II), i.e. $y^{r \pi}(S)=\widehat{C}(S)$. Since $y^{r \pi} \in \operatorname{Core}(C)=$ Core $(\widehat{C})$, we have $y^{r \pi}(S) \leq \widehat{C}(S)$. When $y^{r \pi}(S)=C(S)$, we have $\widehat{C}(S) \leq$ $y^{r \pi}(S)$ because $\widehat{C}(S) \leq C(S)$. When $y^{r \pi}(S) \neq C(S)$, under statement (I) there exits $\emptyset \neq T^{\prime} \subset P$ satisfying (I $\left.a\right)$ and (I $b$ ). We now apply the induction hypothesis on statement (II) to deduce that for all $\pi^{a} \in \Pi$ order $t^{*}$-compatible with $T^{\prime}$, and $\pi^{b} \in \Pi$ order $t^{*}$-compatible with $P \backslash T^{\prime}$, we have $\widehat{C}\left(N \backslash T^{\prime}\right)=$ $y^{r \pi^{a}}\left(N \backslash T^{\prime}\right)$ and $\widehat{C}\left(S \cup T^{\prime}\right)=y^{r \pi^{b}}\left(S \cup T^{\prime}\right)$. Under (I $\left.a\right)$ and (Ib), we have $y^{r \pi^{a}}\left(T^{\prime}\right)=y^{r \pi}\left(T^{\prime}\right)$ and $y^{r \pi^{b}}\left(P \backslash T^{\prime}\right)=y^{r \pi}\left(P \backslash T^{\prime}\right)$, respectively. Hence, 


$$
\begin{aligned}
\widehat{C}\left(N \backslash T^{\prime}\right) & =y^{r \pi}\left(N \backslash T^{\prime}\right) \text { and } \widehat{C}\left(S \cup T^{\prime}\right)=y^{r \pi}\left(S \cup T^{\prime}\right) . \text { Thus, } \\
\widehat{C}(S) & =\min \left\{C(S), \min _{\emptyset \neq T^{\prime \prime} \subset P}\left\{\widehat{C}\left(S \cup T^{\prime \prime}\right)+\widehat{C}\left(N \backslash T^{\prime \prime}\right)-C(N)\right\}\right\} \\
& \leq \min _{\emptyset \neq T^{\prime \prime} \subset P}\left\{\widehat{C}\left(S \cup T^{\prime \prime}\right)+\widehat{C}\left(N \backslash T^{\prime \prime}\right)-C(N)\right\} \\
& \leq \widehat{C}\left(S \cup T^{\prime}\right)+\widehat{C}\left(N \backslash T^{\prime}\right)-C(N) \\
& =y^{r \pi}\left(S \cup T^{\prime}\right)+y^{r \pi}\left(N \backslash T^{\prime}\right)-C(N) \\
& =y^{r \pi}\left(S \cup T^{\prime}\right)+y^{r \pi}(N)-y^{r \pi}\left(T^{\prime}\right)-C(N) \\
& =y^{r \pi}(S) .
\end{aligned}
$$

concluding the proof.

\section{A.5 Proposition 3}

We proceed by contradiction. Assume $\operatorname{ExtCore}(C) \neq\left\{y^{r \pi}\right\}_{\pi \in \Pi}$. Since each $y^{r \pi}$ is a core allocation, we have that there exists an extreme core allocation $x$ that does not belong to the convex hull of $\left\{y^{r \pi}\right\}_{\pi \in \Pi}$. From this, we deduce that there exists some $S$ such that $x(S)-y^{r \pi}(S)$ has the same (nonzero) sign for each $\pi \in \Pi$. That is, either $x(S)-y^{r \pi}(S)>0$ for all $\pi \in \Pi$, or $x(S)-y^{r \pi}(S)<0$ for all $\pi \in \Pi$. Notice that $S$ is one of the sets that determine a saturate constraint on a face of the convex hull of $\left\{y^{r \pi}\right\}_{\pi \in \Pi}$. Since $x$ does not belong to this convex hull, then it should lay inside the opposite side of the half-space. We can assume $x(S)-y^{r \pi}(S)>0$ for all $\pi \in \Pi$ because, in the opposite, we instead consider $T=N \backslash S$.

We now take $\pi \in \Pi$ such that $y^{r \pi}(S)=\widehat{C}(S)$. Existence of such a $\pi \in \Pi$ is guaranteed by Proposition 2. Then, $x(S)>\widehat{C}(S)$ and thus, by Lemma 1, $x$ does not belong to Core $(\widehat{C})=\operatorname{Core}(C)$. Hence the contradiction.

\section{A.6 Theorem 2}

We first show that $\operatorname{Core}\left(C^{\text {Pub }}(N, c)\right) \subseteq \operatorname{Core}(C(N, c)) \cap \mathbb{R}_{+}^{N}$. Let $x \in$ Core $\left(C^{P u b}(N, c)\right)$. Then

$$
x(S) \leq \min _{T \subseteq N \backslash S} C(S \cup T, c) \leq C(S, c)
$$

for all $S \subseteq N$, so $x \in \operatorname{Core}(C(N, c))$. It remains to show that $x \in \mathbb{R}_{+}^{N}$. Suppose that $x_{i}<0$ for some $i \in N$. Since $C^{P u b}$ is monotonically increasing, 
we have that $C(N \backslash\{i\}, c) \leq C(N)$ and thus $x(N \backslash\{i\})>C(N \backslash\{i\}, c)$, contradicting the assumption that $x \in \operatorname{Core}\left(C^{P u b}(N, c)\right)$.

We next show that $\operatorname{Core}(C(N, c)) \cap \mathbb{R}_{+}^{N} \subseteq \operatorname{Core}\left(C^{P u b}(N, c)\right)$. Let $x \in$ $\operatorname{Core}(C(N, c)) \cap \mathbb{R}_{+}^{N}$. Since $x \in \operatorname{Core}(C(N, c))$, we have, for any $S \subset N$ and $T \subseteq N \backslash S$, that

$$
x(S \cup T) \leq C(S \cup T, c)
$$

which, with budget balance, implies that

$$
x(N \backslash S)-x(T) \geq C(N, c)-C(S \cup T, c) .
$$

This inequality can be rewritten as

$$
x(N \backslash S) \geq x(T)+C(N, c)-C(S \cup T, c) \geq C(N, c)-C(S \cup T, c)
$$

where the last inequality comes from the fact that $x \in \mathbb{R}_{+}^{N}$. Under budget balance, $x(S) \leq C(S \cup T, c)$. Since this inequality was obtained for an arbitrary $T \subseteq N \backslash S$, we obtain $x(S) \leq \min _{T \subseteq N \backslash S} C(S \cup T, c)$, which is the condition for $\operatorname{Core}\left(C^{P u b}(N, c)\right)$.

\section{A.7 Proposition 5}

Observe that for all $\pi \in \Pi^{*}(c)$ and all $S \in N \backslash P^{\pi i+1}$, we have

$$
C\left(S, P^{\pi i+1}, y^{r \pi}, c\right)=C(S, c) .
$$

Therefore, for all $\pi \in \Pi^{*}(c)$ and all $i=1 \ldots, n$, we have

$$
\begin{aligned}
y_{\pi_{k}}^{r \pi}(C) & =C\left(\left\{\pi_{1}, \ldots, \pi_{j}\right\}, c\right)-C\left(\left\{\pi_{1}, \ldots, \pi_{j-1}\right\}, c\right) \\
& =\min _{k=0, \ldots, j-1} c_{\pi_{k} \pi_{j}} \\
& =y_{\pi_{j}}^{b \pi}(C)
\end{aligned}
$$

Hence, $y^{b \pi}(C)=y^{r \pi}(C)$.

\section{A.8 Proposition 6}

Trudeau (2012) showed that for elementary problems, Core $(C)=\operatorname{Core}\left(C^{* *}\right)$ and that $\left(N, C^{* *}\right)$ is concave. By the properties of the Shapley value for concave games, $y^{c c}$ is the average over the set of permutations of incremental cost allocations, with each of them being an extreme core allocation. It is obvious that the incremental cost vector corresponding to order $\pi$ is exactly $y^{r \pi}$. We thus have that $y^{s}(C)=y^{c c}$. 


\section{A.9 Proposition 7}

Consider a connected component $T \subseteq N_{0}$. If $0 \in T$, then $C(T \backslash\{0\})=0$. Otherwise, $C(T \backslash\{0\})=1$. In such a case, $c^{*}$ is such that $c_{i j}^{*}=0$ for all $i, j \in T$. By the properties of the public mcst problem, we must have that $C^{P u b}(S \backslash\{0\})=C^{P u b}(T \backslash\{0\})$ for all $S \subseteq T$. Therefore, in both cases, the changes are identical.

We next show that there are no other changes. If $i, j$ belong to different connected components, say $T_{1}$ and $T_{2}$, then $c_{i j}^{*}=c_{i j}$. We also have that for all $R \subseteq T_{1}$ and $S \subseteq T_{2}, C^{P u b}(R \cup S)=C^{P u b}(R)+C^{P u b}(S)$. Therefore, we do not need to make any other changes.

After the modifications, to obtain $y^{f}$ we take the Shapley value of $\left(N, C^{*}\right)$, the cost game associated with $c^{*}$. Given that $\left(N, C^{*}\right)$ is concave (Bergantiños and Vidal-Puga, 2007), it is immediate that $y^{s}\left(C^{P u b}\right)=y^{f}$.

\section{B Appendix - Counter-examples for some prop- erties}

In this section we show that known results for similar methods cannot be applied for mcst problems. We use the following example:

Example 6 Let $N=\{1,2,3,4\}$ and $c$ be as described in the following ( $i$ horizontally, $j$ vertically) and illustrated in Figure 3:

$\begin{array}{ccccc}c_{i j} & 1 & 2 & 3 & 4 \\ 0 & 5 & 7 & 8 & 9 \\ 1 & & 7 & 7 & 5 \\ 2 & & & 4 & 2 \\ 3 & & & & 5\end{array}$

The unique mcst uses edges $(0,1),(1,4),(2,4)$ and $(2,3)$, for a total cost of 16 .

Our method provides 10 distinct extreme points of the core: $(3,1,5,7)$, $(3,1,8,4),(3,2,4,7),(3,3,8,2),(3,7,4,2),(4,1,8,3),(5,1,7,3),(5,2,4,5)$, $(5,4,7,0)$ and $(5,7,4,0)$.

\section{B.1 Almost-concavity}

Núñez and Rafels (1998) prove that almost-concavity is a sufficient condition for the set $\left\{y^{r \pi}\right\}_{\pi \in \Pi}$ to be the set of extreme points of the core. Almost- 


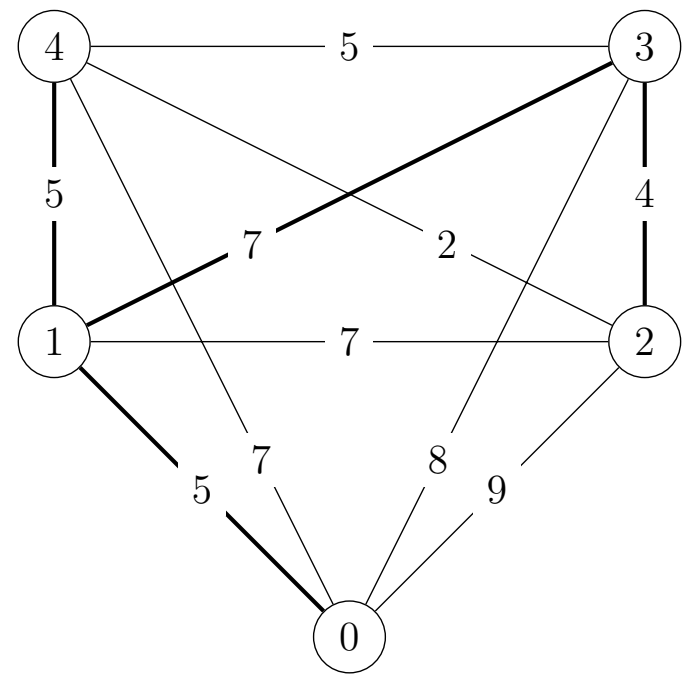

Figure 3: Example of a minimal cost spanning tree problem.

concavity is defined as follows: $C(S)+C(T) \geq C(S \cup T)+C(S \cap T)$ for all $S, T \subset N$ such that $S \cup T \neq N$.

In our example, it is easy to see that $C(\{3\})=8, C(\{1,3\})=12, C(\{2,3\})=$ 11 and $C(\{1,2,3\})=16$. We thus have that $C(\{1,3\})+C(\{2,3\})<$ $C(\{1,2,3\})+C(\{3\})$, which contradicts the almost-concavity condition.

\section{B.2 Concavity of the corresponding exact game}

It is known that for each balanced game there exists a unique exact game with the same core as the original game. By construction, in our case, that exact game is $\widehat{C}$. As shown by Funaki et al. (2007), a sufficient condition for the lexicore to be equal to the core is for that exact game to be concave. The condition for concavity is $C(S)+C(T) \geq C(S \cup T)+C(S \cap T)$ for all $S, T \subset N$.

We go back to our example. It is easy to see that $\widehat{C}(\{3\})=8, \widehat{C}(\{1,3\})=$ $12, \widehat{C}(\{2,3\})=11$ and $\widehat{C}(\{1,2,3\})=16$. We thus have that $\widehat{C}(\{1,3\})+$ $\widehat{C}(\{2,3\})<\widehat{C}(\{1,2,3\})+\widehat{C}(\{3\})$, which contradicts the concavity condition. 


\section{B.3 The CoMa property}

The marginal vector of $C$ with respect to order $\pi$ is defined as followed. For all $i=1, \ldots, n, m_{\pi_{i}}^{\pi}=C\left(\pi_{1}, \ldots, \pi_{i}\right)-C\left(\pi_{1}, \ldots, \pi_{i-1}\right)$.

A game $C$ has the CoMa property if $\operatorname{ExtCore}(C) \subseteq\left\{m^{\pi}\right\}_{\pi \in \Pi}$. i.e. all extreme core allocations are also marginal vectors.

Núñez and Rafels (2003) show that the assignment problem, which shares many characteristics of the mcst problem, has the CoMa property. We show that it is not the case for most problems.

In our example, the marginal vectors are $(1,1,5,9),(1,2,4,9),(2,1,4,9)$, $(2,1,8,5),(3,0,4,9),(3,0,8,5),(3,3,8,2,(3,7,4,2),(4,1,8,3),(4,4,8,0)$, $(5,1,7,3),(5,2,4,5),(5,3,3,5),(5,3,8,0),(5,4,7,0)$ and $(5,7,4,0)$.

We thus have that $(3,1,5,7),(3,1,8,4)$ and $(3,2,4,7)$ are extreme points of the core but not marginal vectors.

\section{B.4 The public game}

It is easy that in our example the public and the private game are the same, i.e. $C(S, c)=C^{P u b}(S, c)$ for all $S \subseteq N$. Thus, all examples in this section carry over to the public game. 